\title{
Linx
}

Revue des linguistes de l'université Paris X Nanterre

$82 \mid 2021$

Entre vieillissement et innovation : le changement linguistique

\section{La vie des mots n'est pas un long fleuve tranquille}

The lifecycle of a word is no bed of roses

Jean-François Sablayrolles

\section{(2) OpenEdition}

Journals

Édition électronique

URL : https://journals.openedition.org/linx/8020

DOI : 10.4000/linx.8020

ISSN : 2118-9692

Éditeur

Presses universitaires de Paris Nanterre

Référence électronique

Jean-François Sablayrolles, «La vie des mots n'est pas un long fleuve tranquille », Linx [En ligne], 82 2021, mis en ligne le 15 juillet 2021, consulté le 20 juillet 2021. URL : http://journals.openedition.org/ linx/8020; DOI : https://doi.org/10.4000/linx.8020

Ce document a été généré automatiquement le 20 juillet 2021.

Département de Sciences du langage, Université Paris Ouest 


\section{La vie des mots n'est pas un long fleuve tranquille}

The lifecycle of a word is no bed of roses

Jean-François Sablayrolles

\section{Introduction}

1 Il est en général plus souvent question des innovations que des obsolescences et disparitions. Le neuf présente ce qu'on nomme l'attrait de la nouveauté, alors que l'ancien, le vieux, paraît moins séduisant (sauf dans certains domaines comme les antiquités, les objets d'art, etc.). Il en va de même pour les langues et les sciences du langage, où l'intérêt pour le changement porte surtout sur ce qui apparaît plus que sur ce qui disparaît, même s'il y a parfois des projets, comme en témoigne le présent volume, qui s'intéressent aussi à ce dernier point.

2 L'incontestable évolution des langues (qui aboutit parfois à leur diversification comme les langues romanes à partir du latin) et l'intérêt pour les sciences historiques et les théories de l'évolution ont conduit à l'émergence de la linguistique historique et comparée au $19^{\mathrm{e}}$ siècle, avant la « révolution » saussurienne, qui a prôné un retour des études linguistiques à la synchronie. La mutation de la linguistique en sciences du langage, dans les années $1980^{1}$, a réouvert des portes, en particulier celle de l'étude de l'évolution des langues, comme en atteste, par exemple, le succès actuel (et assez récent) du concept de grammaticalisation dû à Antoine Meillet au tout début du $20^{\mathrm{e}}$ siècle.

Dans cette optique d'un retour à l'histoire, la métaphore biologique ancienne de la vie des langues et de la vie des mots reste prégnante, voire se renforce, malgré ses nombreuses inadéquations. Par ailleurs l'âge des mots, si l'on peut dire, ne joue quasiment aucun rôle dans les productions langagières des locuteurs à la notable exception des néologismes et des archaïsmes qui seront interrogés du point de vue de 
leur inscription dans le temps qui passe, avant que ne soit brièvement évoquée la manière dont ces derniers et les mots disparus peuvent être repérés.

\section{Inadéquation de la métaphore biologique}

Que ce soit pour les mots ou que ce soit pour les langues, la métaphore biologique comme un organisme vivant qui naît, vit et meurt est très répandue et bien installée dans la durée, au point de constituer une évidence peu remise en question, avec même probablement souvent perte du caractère métaphorique et prise au pied de la lettre de cette formulation ${ }^{2}$. Cette conception comporte de nombreuses failles.

\subsection{La prétendue vie de mots, sans naissance et sans mort}

Pour que la métaphore biologique fonctionnât, il faudrait que tous les mots et toutes les langues naissent, vivent et meurent. C'est loin d'être le cas, et les contre-exemples sont trop nombreux pour constituer des exceptions qui confirmeraient la règle.

\subsubsection{Les mots hérités}

6 En lexicologie, le terme vieillissement s'intègre souvent dans la métaphore biologique, utilisée, entre autres, par Arsène Darmesteter à la fin du $19^{\mathrm{e}}$ siècle (1887), comme une étape de la vie d'un mot, entre sa naissance, sa maturité et sa mort. Cette conception "biologique ", déjà critiquée par ses contemporains (Bréal [1887] 1897 et $2005: 191^{3}$, Meillet [1905: 234] $2015: 312$ ) est de fait inadéquate. On ne peut pas, en effet, assigner de date de naissance à des mots hérités, qui préexistent à l'apparition d'une langue comme telle (le français dans sa variante hexagonale en l'occurrence pour cette contribution) et dont l'existence de certains, que l'on reconstruit, est supposée dès l'indo-européen (père, mère, os, etc. ${ }^{4}$ ). Un certain nombre de mots n'ont ainsi pas cessé d'être utilisés depuis des millénaires, et leurs évolutions sémantiques ainsi que leurs modifications phonétiques progressives et insensibles pour les locuteurs qui les déformaient involontairement et inconsciemment, ne les rendent pas nouveaux. Il y a donc continuité, aussi loin qu'on puisse remonter, sans qu'on puisse atteindre une origine première. En tout état de cause, ces mots hérités ne sont pas nés dans les langues modernes où ils circulent encore, et circuleront encore probablement longtemps. Il faut bien prendre garde que la date de leur première attestation écrite dans des documents, telle qu'elle est fournie dans des dictionnaires généraux, historiques ou étymologiques, ne correspond pas à leur surgissement dans la langue, qui est avant tout orale.

\subsubsection{Les mots éternels et les mots sans date de décès}

7 Il est très risqué de faire des prédictions. C'est particulièrement vrai en linguistique où celles d'éminents linguistes, comme Ferdinand Brunot (1905-1943) ou Albert Dauzat (1943), ont été démenties par les faits (l'impossibilité de la résurrection des superlatifs en -issime ou celle de la création de entrisme par exemple ${ }^{5}$ ). La mort de beaucoup de mots ne peut guère être fixée qu'aux calendes grecques (la mort de père, etc. est-elle 
envisageable et prédictible ?). Les mots fréquents sont très souvent des mots anciens, dont la disparition de l'usage paraît hautement improbable parce qu'ils correspondent à des référents incontournables dans la vie des communautés humaines. Les racines indo-européennes pour signifier l'élan vital / la jeunesse ( ${ }^{*}$ yeu $)$ et la mort $\left({ }^{*} \mathrm{me} / \mathrm{or}\right)$ sont ainsi encore présentes dans beaucoup de mots des langues modernes (dont jeune et mort en français, young en anglais et jung en allemand pour le premier), même si elles ne sont pas toujours immédiatement reconnaissables. Il arrive néanmoins que des remplacements éliminent, progressivement, certains de ces mots fondamentaux. Manger vient de manduco, as, are " mâcher » qui s'est substitué à edo, is, edere ou esse. Le recours à des images concrètes (la mastication) ou à des paradigmes plus réguliers morphologiquement que les mots irréguliers ou devenus tels, et moins touchés aussi par l'usure phonétique que ces mots anciens et hérités ont subie, explique ces substitutions et la disparition de mots à l'utilité pourtant incontestable.

8 Mais, même dans ces cas de disparition de mots, le recours à la métaphore biologique ne convient pas. La mort d'un animé ou d'un végétal est en effet un événement ponctuel, même précédé d'une longue agonie. Or, pour les mots dont on se rend compte, a posteriori, qu'ils ont disparu de l'usage, on peut tout au plus indiquer une période de diminution de leur utilisation, mais pas de date précise de décès, comme on peut le faire pour les êtres animés ${ }^{6}$.

On ne peut pas faire non plus que ces mots n'aient pas existé et n'aient pas circulé (comme c'est aussi le cas pour les êtres vivants). Ceux qui ont des attestations écrites perdurent même dans les énoncés où ils figurent et où des lecteurs les découvrent ou les reconnaissent, du moins dans leur compétence passive. Ce sont des mots dormants, comme la Belle au bois dormant qu'un lecteur - et non un prince charmant - vient réveiller (mais pas, au sens propre, ressusciter).

\subsection{La prétendue vie de langues}

10 Comme pour les mots, la métaphore biologique appliquée aux langues ne convient pas.

\subsubsection{La mort des langues, parfois datable}

11 On peut néanmoins dater assez précisément la mort de certaines langues, quand le dernier locuteur natif d'une langue passe de vie à trépas. Elles ne peuvent au mieux subsister que comme " langues mortes", si elles ont laissé des traces écrites ou ont été décrites, mais la plupart ont disparu corps et bien, sans laisser aucune trace. Certaines langues de culture, qui ont disparu comme langues vivantes, sont néanmoins susceptibles d'utilisations comme le latin scolastique et actuellement le latin d'Église, voire de résurrection comme langue vivante: c'est le cas de l'hébreu moderne. Le nombre de langues complètement disparues est sans doute plus grand que celui de celles qui se parlent, et qui ne cessent de diminuer. Chaque année plusieurs langues disparaissent. D'après des chiffres de l'Unesco ${ }^{7}, 3,85 \%$ des quelque 7000 langues parlées sur le globe ont disparu depuis 1950 ; près de $10 \%$ sont dans une situation critique, et près de $10 \%$ sont aussi considérées comme sérieusement en danger.

La plupart des autres langues évoluent plus ou moins rapidement et plus ou moins profondément. Dans certains cas extrêmes, et sur la longue durée, elles se diversifient en plusieurs langues, sans qu'une date de décès puisse être indiquée précisément. 


\subsubsection{Pas de date de naissance des langues naturelles} naturelle, à la différence de langues artificielles comme l'espéranto (le 26 juillet 1887), l'interlingua (1924, mais sa mise au point s'est étalée sur trois décennies), etc. Il s'agit de continuum dans l'évolution : les langues romanes se sont peu à peu différenciées de la langue source commune (le latin) et se sont singularisées, comme l'ont fait, bien avant elles, les langues indo-européennes issues d'une langue antérieure dont on n'a pas d'attestation matérielle, mais dont la comparaison des langues qui en sont issues conduit à postuler l'existence et permet de reconstituer certains traits. Mais il serait illusoire de penser qu'on peut recréer complètement la langue disparue. On en veut pour preuve la reconstitution du latin que les langues romanes permettraient si celui-ci avait complètement disparu : le morphème grammatical $u t$, polyvalent et très fréquent, ne serait pas restitué car il a disparu sans laisser de trace dans les langues « filles ».

Et ces dénominations de langue mère, fille, sœur, fondées elles-aussi sur la métaphore biologique et les liens de parenté entre êtres animés, sont en fait également impropres, malgré leur commodité, du fait de l'absence de rupture comme l'est une naissance. La continuité qui prévaut au sein des évolutions ne permet pas de "faire sortir » une langue d'une autre comme lors d'un accouchement.

\section{Le temps ne fait rien à l'affaire}

En outre l'âge des mots ne fait rien à l'affaire dans les énoncés produits : on fait se côtoyer des mots de dates très diverses, hérités, créés ou empruntés à des époques variées, sans que les locuteurs aient nullement conscience de ces disparités chronologiques. Quelques cas font néanmoins exception de ce point de vue: les néologismes et les archaïsmes en particulier.

\subsection{Néologisme et temporalité}

\subsubsection{Possibilités variables de datation de la naissance des néologismes}

On peut parfois dater l'apparition d'un néologisme, alors qu'il n'est encore qu'un hapax avant son éventuelle diffusion. Ainsi inénervable a été créé le 3 novembre 2009, devant l'Assemblée nationale, par François Fillon, alors premier ministre. François Bayrou lui a emboîté le pas le 9 juin 2010 en se déclarant ininstrumentalisable. Michel Arrivé ([2005] 2010) donne aussi quelques exemples de verbes dont on connaît le créateur et les circonstances d'énonciation (ainsi de "désembouler le sapin » créé par une petite fille après des fêtes de Noël, le 5 janvier 2002). Mais il faut reconnaître avec lui que ce n'est pas le cas le plus fréquent et souvent on saisit un mot "nouveau» au début de sa circulation, sans qu'il soit toujours possible de remonter à la source, à la première attestation - comme le groupe Neoveille en fait régulièrement l'expérience dans ses réunions de travail sur la plateforme qui porte ce nom ${ }^{8}$ - sans compter les créations multiples indépendantes, toujours possibles. Rien n'empêche en effet plusieurs locuteurs de créer le même mot, indépendamment les uns des autres, de manière quasi simultanée ou à des moments différents. On ne peut que rarement avoir de certitude à ce sujet sauf quand des remarques épilinguistiques accompagnent la création donnée 
comme telle. Mais, même dans ce cas, il peut y avoir des erreurs. Ainsi un internaute s'est dit surpris, il y a un certain nombre d'années déjà, de voir circuler le verbe comater " être dans un état de quasi-somnolence » qu'il pensait avoir créé et être seul à utiliser. Il arrive souvent qu'on soit amené à attribuer la paternité d'une création que l'on repère à des entités anonymes collectives : on peut, au mieux, identifier un groupe, plus ou moins large, où apparaissent tels ou tels néologismes sans qu'il soit possible d'assigner la responsabilité de leur émergence à un individu particulier, même si, de fait, il $\mathrm{y}$ a eu un premier emploi imputable à un individu (ou à plusieurs simultanément), mais il est inatteignable.

\subsubsection{La fin de la néologicité}

18 Quant à l'avenir d'une création, elle est souvent imprévisible. Il faut de ce point de vue bien différencier la date de première attestation repérable (qui peut parfois remonter dans le temps, avec la découverte d'autres documents, plus anciens) de la période de diffusion, qui peut être plus tardive, parfois de beaucoup. Le Petit Robert indique dans certains cas ce décalage temporel entre les deux ${ }^{9}$.

19 Certaines créations sont, pour ainsi dire, mort-nées. La tentative de faire exister quelque chose en lui donnant un nom, comme par un acte de baptême, n'est pas nécessairement couronnée de succès. Ainsi le nouveau naturel créé par Télérama pour un type de films français du milieu des années 1970, a fait long feu, comme l'ont d'ailleurs reconnu les critiques de cet hebdomadaire quelques années plus tard. Ces cas recouvrent ce que Pascaline Dury et Patrick Drouin (2010) nomment nécrologismes, qu'ils traquent avec des outils informatiques appropriés dans des domaines de spécialités.

20 D'autres créations se diffusent plus ou moins rapidement et largement selon le nombre et l'importance des classes sociales qui les utilisent, au point de pouvoir perdre leur néologicité et d'être alors lexicographiés ${ }^{10}$. C'est alors l'enterrement de la néologicité, et non du mot. Mais cette perte de statut s'étale dans le temps, et n'est pas ponctuelle comme une cérémonie de baptême ou une cérémonie funéraire.

21 D'autres créations encore disparaissent après un temps plus ou moins long, pour diverses raisons. Une première est la disparition des référents dénommés, comme le vocabulaire des fiches perforées avec l'avènement de l'informatique. Il en va de même du minitel, qui a été nouveau il n'y a pas si longtemps, et qui est maintenant complètement désuet, et même inconnu des générations les plus jeunes. L'évolution des matériels de reprographie a remisé aux oubliettes les machines à boule (IBM, de 1961), les imprimantes à marguerite, le papier listing ou papier continu avec des bandes Caroll (percées de trous pour les roues à picot de l'imprimante), et détachables après impression, etc.

22 Une autre raison de la disparition de mots est leur remplacement par d'autres mots du fait de l'évolution des mentalités et du politiquement correct. Ainsi l'infamant fille-mère a été supplanté par mère célibataire, ou foyer monoparental ${ }^{11}$. Des phénomènes de mode, aux motivations variées et souvent floues, conduisent aussi à des substitutions partielles (entraîneur supplanté par coach ; parrain, soutien, mécène par sponsor), ou du moins privilégient certains items au détriment d'autres qui prennent un coup de vieux : soulier a été quasiment éliminé par chaussure, du moins en français hexagonal. 
23 En revanche, on indique rarement l'éternelle jeunesse de certaines créations, qui ne sont pas du tout menacées de vieillissement: la plupart des néologismes littéraires, comme délocher de Rabelais, sont encore perçus comme tels par les lecteurs des siècles postérieurs, du moins pour des créations qui sont restées des hapax. Mais des mots récents à l'époque de l'écriture et ressentis par les contemporains comme des néologismes perdent ce statut pour des lecteurs de générations postérieures si les mots se sont diffusés ensuite.

\subsection{L'archaïsme}

24 Le concept bien connu d'archaïsme est en fait peu et mal défini, et employé pour des réalités hétérogènes qu'il faut distinguer avant de s'interroger sur la manière de leur repérage manuel et de leur extraction semi-automatique.

\subsubsection{Archaïsme et paléologisme}

L'archaïsme, présenté ordinairement comme antonyme de néologisme, a été parfois, étonnamment, assimilé à cette notion («les meilleurs des néologismes sont les archaïsmes » Brunot, HLF tome XII, livre V, ch. 2, p. 103). Ce paradoxe vient d'une confusion entre les mots (et expressions) sentis comme vieillis, datés (à toute biture, ça biche, cibiche, poitrinaire, vache "sacoche", etc.) et des mots réintroduits après avoir disparu (décisionnaire, vagissement) : ceux-ci seuls, du fait de l'absence d'une charge sémantique disponible dans la mémoire des locuteurs et interlocuteurs et de leur absence de connotation, produisent le même effet que les néologismes. J'ai proposé de les nommer paléologismes (voir Sablayrolles 2000 : 191-193, 2010, 2018 et 2019 : 206). En revanche les mots archaïques sont connus des membres de la communauté linguistique - du moins d'un certain nombre d'entre eux - et le phénomène générationnel joue à plein : des mots connus mais non employés par des personnes d'un certain âge ne sont même plus connus par des plus jeunes. Pour ceux qui les connaissent encore, ces mots semblent désuets et leur rappellent leur jeunesse, lorsque des personnes plus âgées qu'eux les employaient naturellement. Ainsi gommeux "prétentieux » ${ }^{12}$, poitrinaire "atteint de tuberculose pulmonaire», etc. étaient courants mais ne le sont plus actuellement et leur emploi est connoté «vieilli ». Le PR indique vx pour le premier et vieilli pour le second.

\subsubsection{Des mots trompeurs : des sens anciens non perçus comme tels}

Des lecteurs non avertis et pas sur leur garde peuvent être victimes d'anachronismes en attribuant à des mots du passé leur sens actuel, alors qu'il était autre à l'époque de l'écriture : les camions qui apportent les denrées aux halles dans Le ventre de Paris de Zola n'ont rien à voir avec nos poids lourds contemporains : ce sont des chariots destinés à transporter de lourdes charges, comme c'est d'ailleurs indiqué en note dans les éditions modernes, mais pas dans celles de l'époque. Il s'agit du cas bien décrit par Meillet ([1905-1906] 2015 : 319-322) de la permanence d'un mot alors que la réalité matérielle dénommée a changé, mais la fonction demeure la même.

D'autres mécanismes de contresens sont à l'œuvre quand certaines acceptions de mots ont disparu et que d'autres ont été créées ou quand ne demeure que le sens premier d'un mot et que s'est effacé un emploi imagé. Des collégiens s'esclaffaient lorsque, dans 
Le Misanthrope de Molière, Alceste poussé à bout par Oronte finit par lui déclarer que son sonnet est bon à mettre au cabinet, ignorant qu'il s'agit d'un meuble, où le ranger et le cacher et pas du lieu d'aisance (sens bien connu des collégiens, alors que Le PR donne cette acception vieillie). Cette acception n'est attestée à l'écrit qu'un peu plus tard (1690), mais peut-être circulait-elle déjà oralement? En tout état de cause, le sens ancien est méconnu de nombre de nos contemporains, comme celui, imagé, du mot ruelle, pour l'espace compris entre le lit et le mur, dans une chambre, espace où s'installaient des invités pour converser avec leur hôte ou hôtesse à la période classique.

\subsection{3. À la recherche des mots vieillis ou disparus (et parfois retrouvés)}

Il n'est pas facile de faire surgir dans sa mémoire des mots en voie d'obsolescence ou déjà disparus. C'est au hasard de lectures d'ouvrages anciens, d'écoute d'archives, de conversations avec des personnes de générations antérieures qu'on les repère. Une anecdote personnelle est révélatrice à cet égard : ma petite-fille m'a repris quand j'ai dit lange et langer dans l'histoire que je lui lisais : ma fille avait en effet remplacé ces mots désuets par couche et changer, employés maintenant plus couramment. Le livre avait à peine plus de trente ans et les couches jetables étaient déjà largement en usage. Les modifications $d u$ vocabulaire ont eu du retard par rapport à l'évolution des pratiques. Il arrive aussi que des commentaires épilinguistiques de locuteurs indiquent le caractère obsolète d'un mot employé, du fait de la disparition des référents dénommés. Ainsi N. Sansu, député PCF, évoquait-il le 13 février 2019 « ce qu'on aurait appelé un dispensaire il y a quelques années. »

Mais c'est surtout dans des discours familiers marqués d'affectivité où le besoin d'expressivité fait se renouveler les mots et expressions qui s'usent qu'on relève un grand nombre de cas d'obsolescence. Ainsi pour la vitesse sur route, on disait et on dit encore rouler à tombeau ouvert, à fond la caisse, à cent à l'heure ${ }^{13}$, mais à toute blinde, à toute berzingue, à toute vibure, à toute biture ne me semblent plus guère employés, et le dernier comporte la marque 'vieilli', dans Le PR qui ignore l'avant-dernier de cette liste.

Au-delà des phénomènes générationnels déjà évoqués, il faut noter que la perception de l'archaïsme est fluctuante selon les individus, ce qui n'est pas étonnant quand on songe que chacun se crée son propre lexique, différent de celui de tous les autres locuteurs de la langue. Sur ce point les réflexions de Meillet (2015 [1905-1906 : 235] : 313 ) qui indique le " caractère essentiellement discontinu de la transmission du langage $»^{14}$ et celles de Grunig (1985: 89-98) sont fort pertinentes et peut-être pas toujours assez présentes à l'esprit des linguistes : tous les jugements épilinguistiques sont formulés en fonction des savoirs linguistiques intégrés et de l'image qu'on se fait de sa langue.

Outre les intuitions et le hasard des rencontres, les dictionnaires peuvent être mis à contribution dans la quête des mots anciens avec la recherche, dans leurs versions informatisées, de marques comme 'vieux', 'vieilli', 'archaïque'... comme l'expression c'est bath et le nom bikini, mais ces marques d'usage (variables dans leur formulation) ne sont pas systématiques et ne se correspondent pas d'un éditeur à l'autre. Ainsi cabinets « lieux d'aisance » n'est pas marqué vieilli dans le Petit Larousse Illustré (PLI millésime 2008), à la différence du $P R$ (millésime 2020). De fait il ne me semble pas si vieilli que cela, moins en tout cas que les petits coins, mais il est vrai que toilettes le concurrence grandement. Il arrive aussi que des mots soient lexicographiés alors qu'ils sont déjà en grande partie hors d'usage, comme cibiche " cigarette », absent du PLI 1978 et marqué 
«fam. et vieilli » dans le millésime 1991. L'introduction de la marque d'usage a dû suivre de peu l'introduction du mot dans le dictionnaire. Le synonyme tige, marqué «familier » dans le Dictionnaire Hachette et PR et absent du PLI et du Lexis, ne semble plus très employé non plus.

La comparaison de plusieurs éditions d'un même dictionnaire millésimé permet de trouver les mots que les lexicographes ont éliminés de la nomenclature. C'est à ce type de comparaison que se livre Camille Martinez d'un millésime sur l'autre (disparition de botoxé et fracturation dans le millésime 2015 du PLI, de gyropode dans le millésime 2018 du Dictionnaire Hachette). C'est aussi le principe adopté par Jean-Claude Raimbault (2006) ${ }^{15}$ qui compare quatre éditions du PLI : 1906, 1952, 1972, 2002 et qui compte 10170 mots disparus. Quant à Héloïse Neefs (2008), elle compare le Littré avec des "dictionnaires d'usage courant » (sans plus de précision) et arrive au chiffre de 25000 mots qui ont disparu. Mais les mots supprimés ne le sont pas nécessairement pour cause de vieillissement. Onglerie a été très vite ôté du PLI, en 2012, parce que c'était une marque déposée.

Il y a plus longtemps, quatre linguistes (Dubois et alii, 1960) ont comparé deux éditions du PLI distantes d'un peu plus de dix ans (1949 et 1960). Près d'un quart du vocabulaire du dictionnaire a été modifié. Une de leurs conclusions (ibid. : 204) est la disparition d'un certain nombre de mots (5 105 pour 3417 ajouts), surtout dans des domaines spécialisés, plus que dans la langue générale, comme des termes d'activités artisanales comme la meunerie, la marine à voile, ou dans l'agriculture de la France rurale d'avant la guerre, tombées largement en désuétude, du moins dans l'usage général d'un pays qui connaît un exode rural et une urbanisation croissante, au moment de ce que l'on a dénommé ultérieurement " les trente glorieuses " ${ }^{16}$.

Plus près de nous, l'avènement des imprimantes et des photocopieuses a fait en grande partie tomber en désuétude des techniques de reprographie antérieures comme la miméographie, les duplicateurs à alcool, appelés aussi ronéotype, et les stencils.

Une recherche automatisée comparant des corpus similaires distants de plusieurs décennies est aussi envisageable : les mots employés dans le corpus ancien et absents du corpus récent seraient des candidats à un dictionnaire de mots vieillis ou disparus, avant une éventuelle résurrection (fortuite ou volontaire : Pivot (2004) qui propose une liste de cent mots plus ou moins oubliés à remettre en circulation, dont carabistouilles remis depuis en selle par le Président de la République actuel (Macron) - clampin, coquecigrue, etc.).

\section{Conclusion}

L'intérêt pour ce qui disparaît ou est en voie de disparaître dans l'évolution des langues et en particulier, du lexique, devrait être plus permanent, s'appuyer sur de grands corpus d'époques différentes maintenant disponibles, et conduire aussi à s'interroger sur les raisons, multiples, des obsolescences et disparitions. La prise en compte de phénomènes générationnels et celle des évolutions sociétales apporterait sans doute beaucoup dans cette voie. Si l'apparition d'innovations peut être assignée à des individus, dans des circonstances particulières, l'obsolescence et la disparition sont des phénomènes collectifs, dont les membres de la communauté linguistique ne sont guère conscients : on se rend en effet rarement compte qu'on n'utilise plus un mot, une expression, une tournure. Des enquêtes dont le protocole serait à mettre 
soigneusement au point pour éviter des biais d'enquêtes linguistiques indiquant trop clairement l'objet de la recherche et faussant les résultats, pourraient aussi être riches d'enseignement.

\section{BIBLIOGRAPHIE}

ARRIVÉ, M., ([2005] 2010), Verbes sages et verbes fous, [ 1 ère éd. Limoges, Lambert-Lucas], $2^{\text {nde }}$ Paris, Belin.

BRÉAL, M., ([1887 repris dans 1897] 2005), « L'histoire des mots » repris en annexe dans Essai de sémantique, [Paris, Hachette] Limoges, Lambert-Lucas.

BRUNOT, F., ([1905-1943] 1966), Histoire de la langue française, Paris, A. Colin.

CERQUIGLINI, B., (2017), Les mots disparus de Pierre Larousse, Paris, Larousse.

DARMESTETER, A., ([1887] 1950), La vie des mots étudiée dans leurs significations, Paris, Delagrave.

DAUZAT, A., (1943), Le génie de la langue française, Paris, Payot.

DUBOIS, J., GUILBERT, L., MITTERAND, H., PIGNON, J., (1960), « Le mouvement général du vocabulaire français de 49 à 60 d'après un dictionnaire d'usage ", Le français moderne, Paris, avril 1960, p. 86-106 et juillet 1960, p. 196-210.

DURY, P., et DROUIN, P., (2010), «L'obsolescence des termes en langues de spécialité : une étude semi-automatique de la 'nécrologie' en corpus informatisés, appliquée au domaine de l'écologie ", dans C. Heine et J. Engberg (eds.), Reconceptualizing LSP. Online proceedings of the XVII European LSP Symposium 2009, Aarhus 2010, p. 1-11.

MARTINEZ, C., http://guterrien.free.fr/page-de-camille-martinez-club-orthographe-INPG.HTML MARTINEZ, C. et SAJOUS, F., http://redac.univ-tlse2.fr/lexiques/dico.html

MEILLET, A. ([1905-1906] 2015), « Comment les mots changent de sens », [L'année sociologique], Linguistique historique et linguistique générale, Limoges, Lambert-Lucas, p. 308-349.

NEEFS, H., (2008), Les disparus du Littré, Paris, Fayard.

PIVOT, B., (2004), 100 mots à sauver, Paris, Albin Michel.

RAIMBAULT, J.-C., (2006), Les disparus du XXe siècle : Les 10000 mots disparus, les 18000 mots apparus au XXe siècle, Nantes, Éditions du temps.

REY, A., 2008, « Les mots, des immortels ? », dans Préface à H. Neefs, Les disparus du Littré, Paris, Fayard, p. 11-30.

SABLAYROLLES, J.-F., (2000), La néologie en français contemporain, Examen du concept et analyse de productions néologiques récentes, Coll. Lexica mots et dictionnaires, Paris, Champion.

SABLAYROLLES, J.-F., (2010), « Archaïsme : un concept mal défini et des utilisations littéraires contrastées », dans L. Himy-Pieri et S. Macé (éds), Stylistique de l'archaïsme, Collection "Poétique et stylistique", Bordeaux, Presses Universitaires de Bordeaux, p. 43-65. 
SABLAYROLLES, J.-F., (2018), « Néologie et / ou évolution du lexique ? Le cas des innovations sémantiques et celui des archaïsmes », ELAD/SILDA, Revue du CEL, Lyon 3, Hors-série Neolex, https://revues.univ-lyon3.fr/elad-silda/index.php?id=228

SABLAYROLLES, J.-F., (2019), Comprendre la néologie. Conceptions, analyses, emplois, Coll. La Lexicothèque, Limoges, Lambert-Lucas.

\section{NOTES}

1. À la suite des assises nationales de la linguistique de 1981, et de la création subséquente de l'Association des Sciences du Langage (ASL) en 1983.

2. L'opinion de Dauzat (1943 : 117) en apporte une preuve éclatante : « Quand Arsène Darmesteter parlait de la vie et de la mort des mots, il ne s'agissait pas d'une métaphore. Le vieillissement et le trépas des mots ont diverses causes.» Jean-Claude Raimbault (2006: 241) ne pense pas autrement: «Notre français est un organisme bien vivant, des cellules meurent, d'autres naissent. »

3. « [D]e dire que les mots naissent, vivent entre eux et meurent, cela est, n'est-il point vrai ? pure métaphore. Parler de la vie du langage, appeler les langues des organismes vivants, c'est user de figures qui peuvent servir à nous faire mieux comprendre, mais qui, si nous les prenions à la lettre, nous transporteraient en plein rêve. M. Darmesteter ne s'est peut-être pas toujours assez défié de cette sorte de mise en scène. » Dans sa préface à l'ouvrage de Neefs (2008), Alain Rey met en garde contre ce type de métaphore, qui omet de présenter les mots comme des signes, soumis à l'usage, comme de la monnaie.

4. Pour les deux premiers, attestés en grec, en latin, en sanskrit, dans les langues germaniques..., Antoine Meillet (2015: 319) montre l'existence d'une évolution sémantique depuis l'indoeuropéen où ces mots avaient une valeur sociale plus que familiale et c'est la racine *gen qui servait à signifier les relations de paternité et maternité. La racine *H3es " os ", attestée en grec (osteon), latin (os, ossis), sanskrit, (asthi) hittite (hastai), vieux slave (kosti) a subi moins de transformations formelles et surtout sémantiques.

5. « Nous ne pouvons pas dire une voie * piétonnière [...] Malgré la richesse de notre dérivation en erie, nous n'avons créé ni *bièrerie, ni *carterie, ni *valiserie », écrit Dauzat (1943: 74), pour contraster avec l'italien, plus prolixe en suffixés. Tous ces mots ont été créés et circulent. À propos de ce même suffixe -erie, J. Dubois et alii (1960: 98) écrivaient aussi qu'il a cessé d'être formateur, en relation avec la disparition des «petites industries artisanales ». Depuis, il a repris une très grande vigueur. Les mêmes auteurs, (ibid. : 208), indiquent que « la mobilité du langage de la mode, particulièrement sensible dans le domaine vestimentaire, provoque des apparitions qui ne peuvent être qu'éphémères ", en donnant l'exemple de blue-jean. Là encore, la prédiction a été démentie par les faits.

6. À ce sujet Alain Rey (2008: 14) n'écrit-il pas : «La date d'apparition fleurit dans les travaux lexicologiques, étymologiques et les dictionnaires de langue; la 'date de disparition' est tout simplement impensable. »

7. "Atlas des langues en danger» archives, sur le site de l'UNESCO. Les informations sont reprises dans le site collaboratif Wikipedia "Langues en voie de disparition ». Ces sites ont été consultés en octobre 2018.

8. Pour plus d'information, voir le site créé par Emmanuel Cartier: https://tal.lipn.univparis13.fr/neoveille/html/login.php?action=login

9. Voir Sablayrolles (2019: 52) avec les exemples de fiabilité daté du $13^{\mathrm{e}}$ siècle et répandu en 1962 , de francophone et francophonie créés en 1880 mais dont la large diffusion n'intervient que vers 1960 , etc. 
10. Ainsi la récente pandémie a fait se diffuser rapidement et largement le nom de la maladie, masculin ou féminin pour $L e P R, C O V I D$, les verbes préfixés déconfiner et reconfiner, le groupe prépositionnel en distanciel, etc.

11. Ce dernier cas n'est pas genré et peut inclure aussi des hommes, même si, dans les faits, ils sont moins nombreux que les femmes dans cette situation.

12. Ce mot dans cette acception datée de 1842 par Le $P R$ «jeune homme que son élégance excessive et son air prétentieux rendent ridicule » était régulièrement employé par la grandmère de ma femme, jusqu'à la fin des années 1980.

13. Qui date un peu vu les performances des véhicules actuels et la limitation à $130 \mathrm{k} / \mathrm{h}$ sur autoroute en France.

14. Il poursuit : «l'enfant qui apprend à parler ne reçoit pas la langue toute faite : il doit la recréer tout entière à son usage d'après ce qu'il entend autour de lui. »

15. Merci à Emanuel Cartier de m'avoir indiqué ce livre, celui d'Héloïse Neefs (2008) et celui de Bernard Cerquiglini (2017).

16. Plusieurs stades de disparition sont distingués par ces auteurs, p. 196-197 : celle de dérivés de mots encore présents dans le dictionnaire malgré leur déclin, celle d'une acception désuète, l'utilisation de marques d'usage "vx », «vieilli », pour un mot ou une de ses acceptions et enfin la disparition pure et simple du mot.

\section{RÉSUMÉS}

De multiples raisons rendent inadéquate la métaphore biologique de la vie des mots et des langues : les mots hérités n'ont pas de naissance ; on ne peut assigner à un mot une date de mort, les langues naturelles ne surgissent pas soudainement ex nihilo, etc. Sauf les cas des néologismes et des archaïsmes perçus comme tels, ainsi que des paléologismes (mots disparus de l'emploi d'une langue et réintroduits dans celle-ci), les locuteurs font se côtoyer dans leurs énoncés des mots d'âges très divers, sans qu'ils le sachent. Sont enfin évoquées des pistes d'identification des archaïsmes et mots disparus.

There are many reasons why the biological metaphor of the lifecycle of a word and a language is inadequate. French words which come directly from Latin have no 'birth'. Similarly it is not possible to date when a word 'dies'. By the same token, natural languages do not suddenly appear out of nowhere, and so on. With the exception of neologisms and archaisms which are recognized as such, as well as paleologisms (words which disappeared from a language but reintroduced at a later period), speakers muster words of greatly varying ages without being aware of their age. In this paper ways of identifying archaisms and words which have disappeared will be examined.

\section{INDEX}

Mots-clés : archaïsme, néologisme, paléologisme, vie des mots et des langues

Keywords : archaism, neologism, paleologism, word and language lifecycles 
AUTEUR

JEAN-FRANÇOIS SABLAYROLLES

USPC et HTL UMR 7597 